shorter wave-length by the same condition. In this way the whole spectrum is divided into a number of contiguous divisions, or patches, which Dr. Green terms monochromatic.

"Tested with this instrument a normal individual will, as a rule, name six distinct colours (viz. red, orange, yellow, green, blue, violet), and will mark out by means of the shutters about 18 monochromatic patches. Occasionally we come across individuals with a greater power of differentiating hues, to whom, as to Newton, there is a distinct colour between the blue and violet, which Newton called indigo. Such individuals will mark out a greater number of monochromatic patches, from 22 up to 29. The limited number of monochromatic patches which can be marked out in this way is at first surprising when we consider how insensibly one part of the spectrum seems to shade into the next when the whole of the spectrum is looked at. The number and position of the patches present, however, great uniformity from one case to another.'

Being curious to know into what class my own vision would fall on this system, I was glad to be tested by Dr. Green last July. The number of patches proved to be I7, a little short of what Dr. Green lays down in the passage above quoted as normal. The limits of the actual patches were as follows:-

$$
\begin{gathered}
780-635^{\frac{1}{2}}-624-6 \mathrm{r} 2-603-595-586-57^{6}-560-54 \mathrm{I}- \\
5^{2} \mathrm{I}-509-500-489 \frac{1}{2}-477-462-443-426 .
\end{gathered}
$$

Thus in the region of the $\mathrm{D}$ lines a patch including wave-lengths between 595 and 586 did not manifest a difference of colour. The interval between the $D$ lines on the above scale being 0.60 , it appears that my "monochromatic patch" was 15 times this interval.

While it is undoubtedly true that in this way of working no colour-difference was perceptible as the eye travelled backwards and forwards over the patch, my experience with colour discs and other colour-mixing arrangements made me feel certain that under more favourable conditions I could discriminate much smaller differences of wave-length. Special experiments have since proved that I can, in fact, discriminate by colour between points in the spectrum so close together as the two D lines.

In order to compare two colours with advantage it is necessary that each should extend with uniformity over a considerable angular area, and that the two areas should be in close juxtaposition. The requirements of the case are sufficiently met by a colour-box (after Maxwell) such as I described nearly thirty years ago. ${ }^{1}$ In this form of apparatus a second slit, placed at the focus, allows a narrow width of the spectrum to pass; but instead of regarding the transmitted portion with an eye-piece, the eye is brought close to the slit and focussed upon the prism, which thus appears uniformly lighted with such rays as the second slit allows to pass. The light thus presented is, of course, not absolutely homogeneous; it includes a mixture of neighbouring spectrum rays, the degree of purity augmenting as the slits are narrowed. With the aid of a refracting prism of small angle (set perpendicularly to the dispersing prisms) the field of view is divided into two parts, which correspond to any desired colours according to the situation of the two primary slits. For the present purpose these primary slits lie nearly in one straight line, inasmuch as the two spectrum colours to be compared are close together.

In making the observations on sensitiveness, one primary slit, as well as the eye-slit, remains fixed, the position being chosen so as to provide vellow light from the neighbourhood of $\mathrm{D}$. The second slit can be moved as a whole while retaining its width.

The procedure is quite simple. If the colours seen are strongly contrasted, the movable slit is displaced until the difference is moderate. Marks may then be given: $\mathbf{O}$, denoting that the difference is uncertain: $R_{1}$, that it is just distinct in the direction of making the second patch the redder; $G_{1}$, that it is just distinct in the opposite direction. Similarly, $R_{2}, G_{2}$, denote differences in the two directions which are more than distinct, and so on. After each observation worth recording, the position of the movable slit is measured. 1 Nature, r881, vol. xxv., pp. 64-66; "Scientific Papers, " vol. i., p. 543.
See also Nature, August i8, roro.

NO. 2 I 52 , VOL. 85$]$
In this manner, as the result of sets of observations made on several days, it was found that a movement of the second slit through $0.15 \mathrm{~mm}$. was sufficient to carry the variable colour from being distinctly redder than the standard to distinctly greener. We may conclude that the eye is capable of appreciating without fail a difference of situation represented by $0.07 \mathrm{~mm}$.

It remains to interpret the result in terms of wavelengths. By allowing light to enter at the eye-slit, or rather at a narrower slit superposed upon it, a spectrum is formed at the other end the scale of which has to be determined. It appeared that the distance from $\mathrm{D}$ to $\mathrm{E}$ was $7 \mathrm{~mm}$. The difference of wave-length between these lines is 62.3. The perceptible difference is $1 / 100$ of this, corresponding nearly enough to the difference between the $D$ lines. I think $I$ am safe in saying that $I$ could distinguish the colours of the two $D$ lines if favourably presented to the eye.

This degree of sensitiveness, though not higher than I had expected, is a little difficult to reconcile with the monochromatic appearance of a portion of the spectrum fifteen times wider. I suppose that the gradual character of the transition is an obstacle to the recognition of differences. The question of angular magnitude may also enter. No doubt a very small apparent magnitude would be unfavourable. It is possible that in Dr. Green's apparatus an eye-piece of higher power, with a corresponding augmentation in the intrinsic brilliancy of the source of light, would allow of an increase in the number of distinguishable patches. The experiment would be worth a trial.

It will be seen that the existence of " monochromatic patches" in the spectrum is far from meaning that the eye is incapable of making chromatic distinctions within their range. I do not infer from this that the results of the method are without significance. Undoubtedly it is possible by means of it to classify colour-vision, and such a classification cannot be without interest, even if we fail as yet to understand exactly what it means.

\section{THE PROGRESSIVE DISCLOSURE OF THE ENTIRE ATMOSPHERE OF THE SUN 1}

I E soleil auquel est consacrée cette conférence est un magnifique sujet d'etudes. Tous les hommes sentent plus ou moins claireinent que les destinées terrestres sont liées étroitement à celles du soleil, et qu'il est nécessaire de reconnaître sa nature intime, son rayonnement total, ses variations, en un mot son action précise et complète sur notre globe. Notre dépendance vis-à-vis du soleil est absolue, et récemment, elle a été résumée d'une manière simple par un homme politique français, maintenant ministre des finances, auquel je demandais un crédit spécial pour l'observatoire de Meudon que je dirige, et pour les recherches solaires. Il refușait d'abord, en alléguant l'acroissement continu des dépenses publiques. Puis, comme j'insistais, il s'écria: "Vous avez raison, le soleil est notre maître à tous; il est impossible que nous ne fassions pas quelque chose." C'est ainsi que l'observatoire de Meudon a pu joindre à son budget ordinaire une somme supplémentaire, certes peu élevée, mais qui est arrivée au moment opportun, et nous a beaucoup aidés dans les recherches que je vous présente aujourd'hui.

L'étude moderne du soleil exige en effet des installations coûteuses, des appareils compliqués et un personnel spécial apte aussi bien aux observations physiques qu'aux observations astronomiques. Or le soleil luit pour tout le monde et mûrit toutes les moissons; et, à priori, il semble naturel que tous les hommes de la planète apportent leur concours aux recherches solaires. Partant de cette idée, j'ai proposé, il y a quelques années, à la Société astronomique de France une taxe spéciale et générale pour le soleil-et d'ailleurs très minime. Si chaque français, ai-je remarqué, donnait par an un sou, un simple sou pour le soleil, la somme totale serait encore élevée; elle permettrait d'assurer l'enregistrement continu du soleil et de ses variations, non encore réalisé, et donc une connaissance plus approfondie de 1'astre. Mais les taxes nouvelles sont toujours plus nombreuses, et celle-là, bien que très faible et très légitime,

1 Discourse delivared at the Royal Institution of Great Britain on Friday June Iว, r 910 , by Dr. H. Deslandres, Membre de l'Institut 
serait probablement écartée. D'ailleurs, il faut bien le dire, l'homme civilisé actuel, le citadin surtout, s'occupent peu du soleil; ils le regardent moins que l'homme primitif et le sauvage qui n'ont ni montre ni almanach. La réalisation de cette idée est réservée pour la cité future, et pour un état social plus parfait que le nôtre.

Le recours au gouvernement, à la collectivité, est une habitude française. Il vaut mieux comme en Angleterre, faire appel à l'initiative privée, à l'initiative d'hommes éclairés et généreux. C'est ainsi qu'a été fondée la Royal Institution, qui a vu éclore tant de belles découvertes et tant de savants illustres. Ce bel exemple doit être proposé à tous, et on sait qu'il a été largement suivi en Amérique où les plus grands observatoires, et surtout ceux consacrés au soleil, sont dus à de simples particuliers.

En fait, dans les cinquante dernières années, grâce à de grandes découvertes, grâce d̀ l'appui des gouvernements et des Mécènes, l'étude du soleil a pris un développement considérable. Les astronomes ont pu lui donner peu à peu une organisation sérieuse et permanente et même l'étendre à l'atmosphère entière de l'astre, jusqu'alors inaccessible.

La découverte principale sur le soleil est la variation périodique de ses taches noires, variations que subissent aussi les facules brillantes de la surface et l'atmosphère entière très étendue. Le soleil entier a une grande oscillation générale; et, fait plus curieux encore, cette oscillation s'étend à la terre et, tout au moins, à ses éléments magnétiques.

L'extension du phénomène solaire à la terre a une importance capitale; elle implique presque nécessairement une action spéciale, nouvelle, exercée par le soleil sur notre Slobe: elle est la cause première de la grande faveur actuelle des recherches solaires. Après la découverte de Sabine et Lamont sur 1'accord de nos variations magnétiques avec le soleil, la science anglaise a accordé la plus grande attention aux taches du soleil; et la première elle a organisé l'enregistrement photographique des taches et des éléments magnétiques sur plusieurs points du globe, et la concentration de tous ces documents dans un même observatoire qui les relève avec précision. Les travaux d'Ellis et de Maunder sur la question sont bien connus et il convient aussi de rappeler ceux de Lockyer et de Shuster, qui ont reconnu récemment dans les variations des taches des périodes plus grandes et plus petites que la période principale de Ix années.

L'action exercée par le soleil sur la terre est attribuée. généralement aux taches; mais elle peut avoir son origine dans l'atmosphère solaire qui a les mêmes variations; d'où la nécessité d'étudier et de relever avec soin cette atmosphère. Or, depuis près de 20 ans. je me suis attaché à la reconnaissance de l'atmosphère entière du soleil, et je vous présente aujourd'hui les résultats les plus récents, qui ont mis au jour les couches supérieures de cette atmosphère jusqu'ici inexplorées.

Atmosphère des éclipses-au bord solaire extérieur.

L'atmosphère du soleil s'est montrée à l'homme pour la première fois dans les éclipses totales, au bord solaire extéricur. Elle forme alors l'anneau lumineux qui se détache sur le fond du ciel devenu noir, en entourant le disque lunaire, également noir. Elle comprend deux parties distinctes, à partir de la lune et du bord solaire: la chromosphère mince et brillante, de couleur rose, de laquelle se détachent les proéminences également roses, et la couronne, plus pâle mais très étendue. Dans ce qui va suivre, il sera question surtout de la chromosphère et des proéminences.

En temps ordinaire l'anneau lumineux des éclipses est caché par 1'illumination beaucoup plus vive de notre ciel. L'écran qui le masque est lumineux; pour l'écarter, l'astronome anglais Sir Norman Lockyer, a en le premier, en I866, l'idée de recourir au spectre, en admettant, ce qui était probable, que l'atmosphère solaire fût gazeuse. C'était une idée de génie, qui depuis a fait son chemin.

L'éclipse de 1868 montre en effet que les proéminences roses sont constituées en grande partie par l'hydrogène incandescent qui émet les radiations déjà reconnues dans le laboratoire sous l'influence de l'étincelle électrique, et en particulier une raie rouge intense appelée $\mathrm{H}_{a}$. Et, après l'éclipse, Janssen aux Indes, Lockyer en Angleterre, avec NO. 2 I 52 , VOL. 85$]$ le spectroscope et la raie rouge, retrouvent les proéminences et la chromosphère des éclipses. Ce résultat a excité un enthousiasme légitime; car la méthode, à la fois simple et féconde, est employée depuis 40 ans à la reconnaissance journalière de la chromosphère, des positions et des formes des proéminences. Cette étude est même plus captivante que celles des taches; car les proéminences ont les formes les plus variées et les changements les plus rapides. Elles apparaissent à toutes les latitudes, et suivent aussi la période undécennale des taches, la durée du maximum étant, il est vrai, plus longue.

L'étude spectrale du bord solaire, poursuivie en temps ordinaire, ou mieux pendant les éclipses, fait aussi connaître la composition chimique de la chromosphère, et aussi la hauteur minime de chaque vapeur, estimée par la longueur de la raie correspondante dans le spectre.

D'une manière générale, les vapeurs à faible poids atomique et les gaz légers s'élèvent le plus haut; tel est le cas de I'hydrogène et de l'hélium. La raie la plus haute dans ces deux gaz est la raie rouge $\mathrm{H} \alpha$ de l'hydrogène, les autres raies de l'hydrogène ayant des hauteurs et des éclats qui diminuent du rouge à l'ultraviolet.

Mais les plus hautes de toutes sont les raies violettes $H$ et $\mathrm{K}$, très brillantes, qui sont émises par les composés du calcium. Comme le poids atomique et la densité de la vapeur de calcium sont relativement élevées, le fait paraît assez étrange; il est expliqué simplement, d'après les idées de Lockyer, par une dissociation du calcium dans le soleil et l'étincelle de nos laboratoires. Les raies $\mathrm{H}$ et $\mathrm{K}$, à tous égards exceptionnelles, sont très brillantes au bord solaire, et assurent aisément la photographie des proéminences avec les plaques ordinaires.

D'autre part, les vapeurs lourdes, qui sont de beaucoup les plus nombreuses s'élèvent peu dans l'atmosphère, et ne sont aisément visibles que dans les éclipses. Elles forment la couche basse de la chromosphère, relativement fort brillante, appelée couche renversante.

\section{Chromosphère projetée sur le disque, couche moyenne.}

Tels sont les résultats principaux de la méthode LockyerJanssen. Ils sont assurément remarquables, mais, à certains égards. incomplets. Ils ne s'appliquent qu'à la partie de la chromosphère extérieure au bord solaire, et même aux vapeurs légères, et élevées de ce bord. La partie intérieure au bord, ou projetée sur le disque, en projection 5o fois plus étendue, lui échappe. Or cette lacune a été comblee de $\mathrm{I}_{892}$ à $\mathrm{I} 894$ par une méthode absolument générale, qui décèle toutes les vapeurs, lourdes ou légères, et leurs couches successives dans la demie-sphère entière tournée vers la terre.

$\mathrm{Au}$ bord solaire, les raies des vapeurs se détachent brillantes sur le spectre continu de notre ciel; mais, sur le disque, ces raies sont noires, comme on sait, et le spectre continu qui leur sert de fond est celui du soleil lui-même et est beaucoup plus intense. A priori la difficulté paraît beaucoup plus grande.

Or les raies $\mathrm{H}$ et $\mathrm{K}$ du calcium présentent une exception à cette règle, et le fait a été annoncé simultanément en février I 892 par Hale et Deslandres. Ces raies noires sont très larges et même les plus larges du spectre solaire; mais, aux points de la surface où est une facule, elles sont renversées, autrement dit elles offrent en leur centre une raie brillante qui même est double et se détache sur la large raie noire aussi bien que la raie des proéminences au bord extérieur. (Voir la Fig. $\mathrm{I}$, qui montre la raie $\mathrm{K}$ et ses composantes $\mathrm{K}_{\mathrm{rv}}, \mathrm{K}_{\mathrm{2v}}, \mathrm{K}_{3}, \mathrm{~K}_{2 \mathrm{R}}, \mathrm{K}_{\mathrm{rR}}$.)

Le résultat a été obtenu par Hale avec un spectrohéliographe, appareil nouveau, assez complexe, qui isole une radiation avec une seconde fente, et, par le mouvement de cette fente lumineuse, fournit une image monochromatique de l'astre. De mon côté, j'ai employé le simple spectrographe ordinaire et des sections successives, mais en préconisant l'emploi du spectrohéliographe.

Cependant les deux observateurs étaient en désaccord sur un point capital. Hale plaçait les vapeurs ainsi décelées dans la facule même, sous la surface; je les plaçais au contraire au-dessus dans l'itmosphère même. Or le spectrographe ordinaire, qui réunit tous les éléments de la question, permet de la résoudre; il est, à ce poirt de vue, supérieur au spectrohéliographe. 
La raie double $K_{2}$ est brillante non seulement sur les facules, mais sur tous les autres points du disque où elle est, il est vrai, plus faible et plus difficile à distinguer. De plus, au bord, la raie brillante double $\mathrm{K}_{3}$, au bord intérieur, est toujours nette en ce point, et est prolongée à l'extérieur par une raie brillante double identique. (Voir la Fig. 2 ci-contre, figure schématique, qui montre bien l'aspect de la raie double $K_{2}$, au bord du soleil et aussi sur une tache.)

Comme la raie $K_{2}$ extérieure au bord représente par définition la chromosphère, la conclusion est la suivante: L'image de la raie $K_{2}$ avec le spectrohéliographe représente la chromosphère entière de l'astre projetée sur le disque.

D'ailleurs les images du calcium faites à Paris en $x 894$ et qui sont les premières images complètes, montrent des
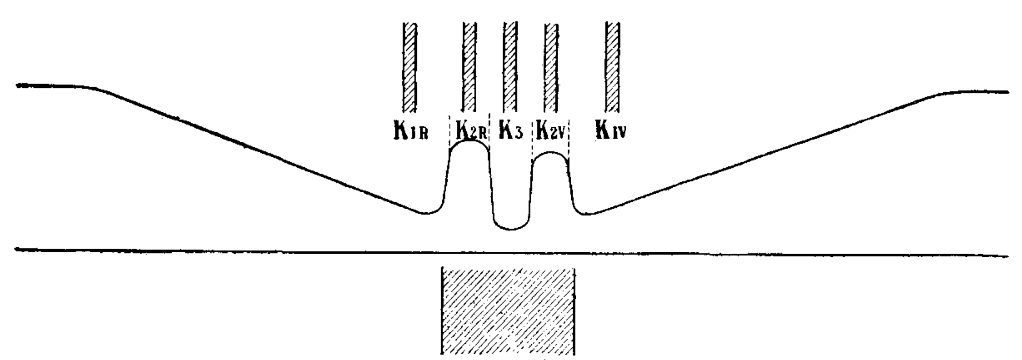

$\mathrm{K} 2.3$

F1G. x.-Courbe des intensités du spectre solaire à l'emplacement de la large raie noire $K$. On a représenté par des traits avec hachures les positions des fentes des spectrohéliographes. rapides. moirs la constitution spéciale de cette raie typique du calcium. Le champ nouveau offert à l'investigation s'annorce comme extrêmement étendu.

\section{Recherches ultérieures. Grand Spectrohéliographe d'un type nouveau.}

Le programme de recherches, indiqué en 1894 , est donc extrêmement vaste. Il a été appliqué en partie dans les années suivantes, et les progrès ont été réels si non très

En 1903, Hale et Ellermann, à l'observatoire Yerkes, reprennent l'étude des raies noires, avec un spectrohélioraphe plus dispersif, et la poursuivent à partir de I906 au Mont Wilson avec des appareils encore plus puissants. Ils ont obtenu de magnifiques images et toute une série de faits nouveaux. Avec les raies de la couche renversante, les résultats sont à peu près les mêmes que ceux de I894; mais les raies de 1'hydrogène, et tout récemment la raie $\mathrm{H}_{\alpha}$ ont montré des phénomènes nouveaux, très curieux, dont il sera question avec détails un peu plus loin.

Cependant la dispersion employée par eux est seulement moyenne; s'ils ont isolé un nombre de raies bien plus grand qu'en 1894 , ils n'ont pas isolé les raies fines; et même dans chaque cas, ils ont isolé la raie entière, ils n'ont pas séparé les parties distinctes de la raie et donc les couches successives de la vapeur. plages faculaires brillantes plus larges que celles de la surface, et aussi les parties brillantes plus petites appelées maintenant flocculi, qui sont présentes aussi bien aux pôles qu'à la lignateur-j'ai vérifié la présence des flocculi aux pôles dans les années de minimum et pendant la période undécennale tout entière.

La raie brillante $\mathrm{K}_{2}$ reste double au bord extérieur jusqu'à 4' ou 5' d'arc, et, comme la chromosphère au bord est haute de ${ }^{\prime \prime} \mathrm{O}^{\prime \prime}$, on peut dire que cette image représente la chromosphère moyenne.

En résumé, si le premier spectrohéliographe ayant donné des résultats a été réalisé en Amérique, c'est en France qu'on a reconnu pour la première fois la chromosphère entière du soleil.

\section{Chromosphère basse.}

Mais on peut aller plus loin. En 1893 , j'ai annoncé que l'isolement d'une raie noire ordinaire avec le spectrohéliographe donnerait l'image même de la vapeur correspondante; et, en I 894 , j'ai isolé avec le petit spectrohéliographe de faible dispersion, organisé à Paris, les bords dégradés de la raie $K$, appelées $\mathrm{K}_{\mathrm{IR}}$, et $\mathrm{K}_{\mathrm{rv}}$, et les raies noires voisines les plus larges de l'aluminium, du fer et du carbone. L'image obtenue differe de celle de $\mathrm{K}_{2}$; les taches masquées par fois avec $K_{2}$ ont toujours leur ombre et pénombre bien nettes, et les plages faculaires sont brillantes au centre comme au bord, mais moins larges que dans l'image $\mathrm{K}_{2}$. En fait, cette image nouvelle est intermédiaire entre l'image de la surface et celle de la couche

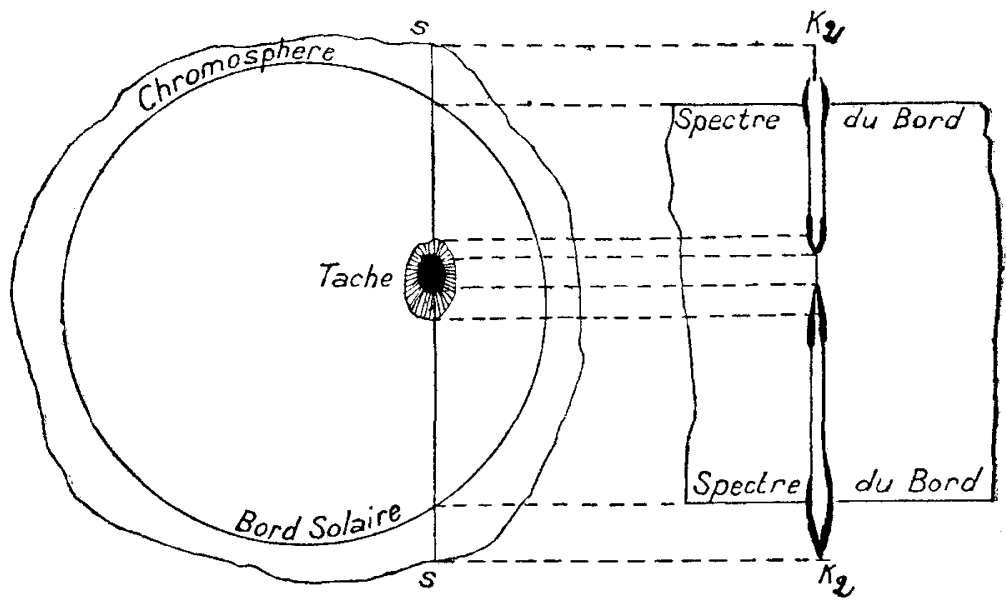

FIG. 2.-(schématique).-ss, section faite par la fente du spectroscope dans le soleil dont la chromosphère et la tache sont très agrandies; $\mathrm{K}_{2}$, raie brillante, attribuée aux vapeurs du calcium, qui apparaît au milieu de la large raie noire $\mathrm{K}$ du spectre normal ; elle est simple et fine au-dessus des taches et à la partie supérieure de la chromosphère, et double sur les autres points, étant alors divisée en deux par la raie noire centrale $\mathrm{K}_{2}$.

Leur image est un mélange de plusieurs images distinctes et de plusieurs couches.

Je me suis proposé de combler cette lacune, et de poursuivre jusqu'au bout le programme de 1894 , en isolant nettement les couches supérieures non encore décelées. Devenu directeur de l'observatoire de Meudon en 1907, j'ai pu diriger de ce côté les ressources de l'observatoire, et, d'autre part la crédit extraordinaire signalé plus haut, nous moyenne chromosphérique $\mathrm{K}_{2}$. Elle représente l'image de la couche renversante entière qui serait obtenue ainsi pour la première fois.

J'ai ajouté qu'une dispersion plus forte permettrait d'isoler les raies plus fines qui sont les plus nombreuses, et, en particulier, la petite raie noire centrale $K_{\text {, }}$ entre les deux composantes $\mathrm{K}_{2}$. Cette raie $\mathrm{K}_{3}$ correspond à la couche supérieure de la chromosphère. La méthode s'annonce ainsi comme absolument générale; elle fournit l'image de toutes les vapeurs solaires, et aussi l'image de leurs couches successives superposées, au moins lorsque la raie est divisible en parties distinctes, ainsi que la large raie $\mathrm{K}$.

Or le nombre des raies solaires s'elève à 20,000; et, d'après Jewell, toutes les raies solaires offrent plus ou NO. 2 I 52 , VOL. 85$]$ 
réalité quatre spectrohéliographes différents réunis autour d'un même collimateur. Le premier est à trois prismes et à deux fentes, avec une chambre de $3 \mathrm{~m}$., et une image du soleil de $85 \mathrm{~mm}$.: le second est à réseau et à deux fentes avec une chambre de même longueur. Le troisième est une disposition différente des deux précédents. Enfin le quatrième, le plus puissant, est à trois fentes, à prismes ou à réseau. Il comprend un premier spectrographe avec chambre de $7 \mathrm{~m}$., ainsi que dans l'appareil classique de Rowland, ce qui permet d'isoler des raies très fines. Mais l'image solaire exigerait une pose trop longue; on la reprend avec une second spectrographe qui le diminue au dégré voulu, et élimine la lumière diffuse intérieure. Le soleil final a un diamètre qui peut être quelconque, et, grâce à certaines dispositions spéciales, il est entier, ce qui n'est pas réalisé dans les autres spectrohéliographes de grande dispersion. Les diamètres habituels sont $6 \mathrm{~cm}$. et $4 \mathrm{~cm}$.

L'appareil, avec ses deux spectrographes, a une longueur totale de i4 m., et, dans ces conditions, reste immobile. $\mathrm{H}$ est même le premier spectrohéliographe dont toutes les parties sont fixes, la plaque étant mise à part. Les pièces mobiles sont la plaque photographique et l'objectif astronomique, qui sont mis en mouvement à la vitesse voulue par des moteurs électriques synchrones et des transformateurs de vitesse spéciaux.

La concordance des mouvements est assurée par des moyens électriques, indépendants de la distance, et le dispositif est présenté comme une solution générale du spectrohéliographe. Chacun des quatre spectrohéliographes a ses avantages particuliers, et le passage de l'un à l'autre se fait en quelques minutes. L'observateur a ainsi à sa disposition des moyens d'investigation variés. D'une manière générale, les spectrohéliographes à deux fentes de $3 \mathrm{~m}$. donnent une image plus grande et plus riche en détails. Le grand appareil de $\mathrm{I} 4 \mathrm{~m}$. à trois fentes, donne, avec une pose plus longue, une image plus petite, mais beaucoup plus pure; il permet d'isoler des raies plus fines.

Les recherches avec cet appareil ont été poursuivies avec un jeune astronome de l'observatoire, M. d'Azambuja, dont le nom est associé au mien.

\section{Révélation de la couche supérieure $K_{3} d u$} Calcium.

En 1908 nous avons pu isoler la petite raie noire centrale $\mathrm{K}_{3}$ du calcium, et donc la couche supérieure de la vapeur. La Fig. I qui montre la raje $\mathrm{K}$ et ses composantes permet de bien juger le progrès réalisé.

Jusqu'alors les spectrohéliographes employés isolaient en même temps l'ensemble des deux composantes brilliantes de $K_{3}$ qui comprennent la raie $\mathrm{K}_{3}$, avec une fente de $\frac{90}{100}$ d'Angström. L'image, appelée par nous image $K_{23}$, était un mélange des couches $\mathrm{K}_{2}$ et $\mathrm{K}_{3}$ avec une prédominance de la couche $K_{2}$, beaucoup plus brillante; la couche supérieure $\mathrm{K}_{3}$ était masquée. Or, avec le grand spectrohéliographe, nous avons pu isoler facilement avec des fentes de $\frac{3}{100}$ d'Angström et plus, isoler soit la raie $\mathbf{K}_{\text {, }}$, soit l'une des composantes de $K_{3}$, et avoir ainsi des images de chaque couche bien pures et exemptes de toute lumière étrangère. Les fentes correspondantes sont indiquées sur la Fig. I par des traits avec hachures.

La vapeur de calcium qui au bord extérieur, s'élève plus que toute les autres, présente ainsi dans l'atmosphère trois couches distinctes superposées. Si on ajoute la surface, on a quatre couches, qu'il est intéressant de comparer.

Lorsqu'on s'élève à partir de la surface, les facules ou plages brillantes de cette surface augmentent progressivement en étendue et en éclat relatif. Les flocculi moyens augmentent aussi, alorsque les petits disparaissent ou sont

NO. 2 I 52 , VOL. 85$]$

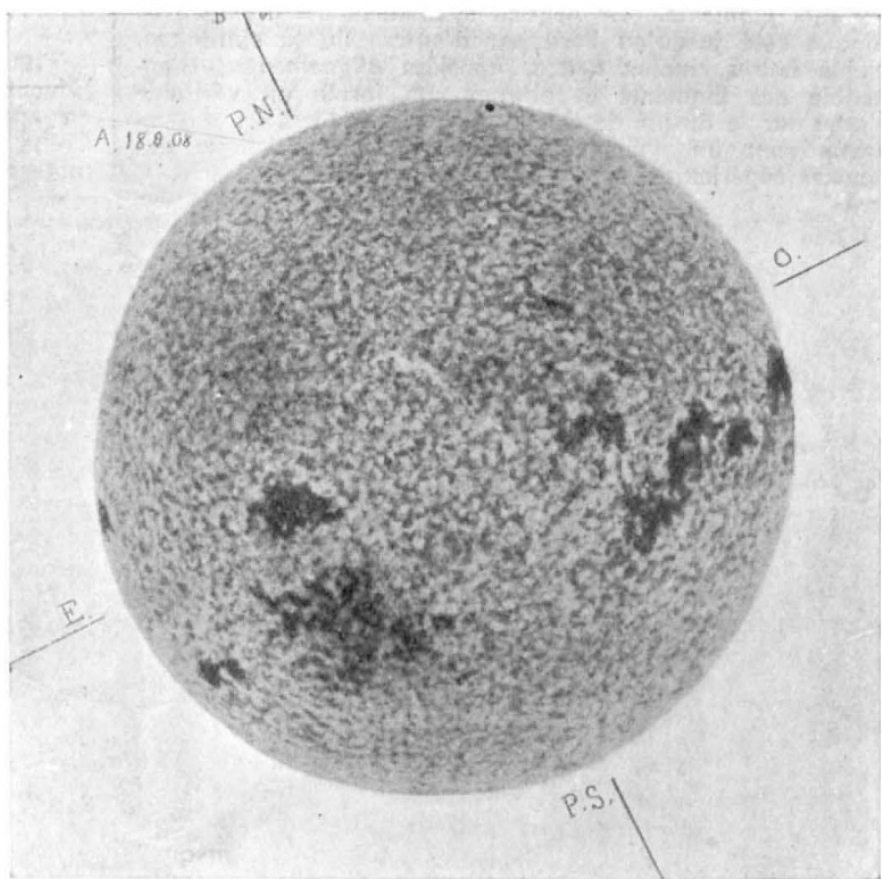

Couche supérieure $\mathrm{K}_{3}$ du calcium.

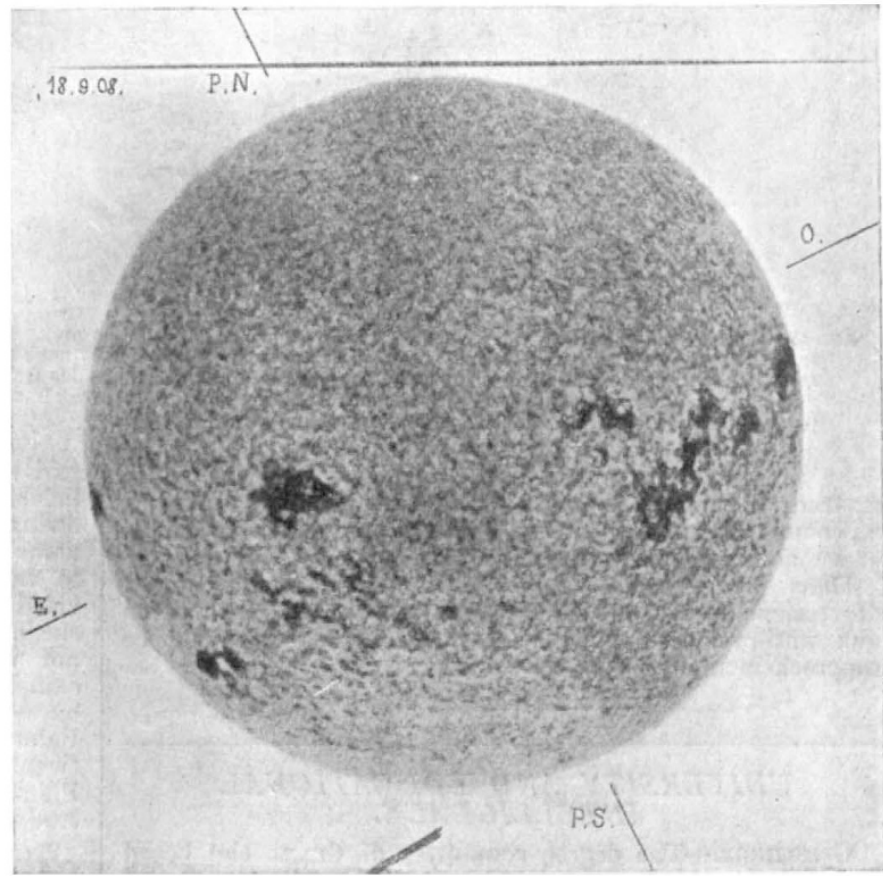

Couche moyenne $\mathrm{K}_{2} \mathrm{du}$ calcium.

Plate I.-Images du is septembre, Ig08.

de flocculi, appelé par moi en I894 rtseau chromosphérique, et formé souvent, sur une étendue notable, de polygones juxtaposés par leurs côtés et leurs sommets, est en général plus net dans la couche supérieure. 
D'autre part les taches noires, qui sont le caractère principal de la surface, diminuent progressivement, lorsqu'on s'élève et même disparaissent.

Par contre apparaissent des lignes noires, invisibles dans les couches basses, lignes souvent très longues et appelées par moi filaments. En général le filament est prolongé de chaque côté jusqu'au bord par d'autres lignes similaires, moins noires, moins nettes, appelées alignements. L'ensemble des filaments et alignements forme un véritable réseau sur le disque de soleil. Les filaments et les alignements sont un phénomène nouveau, caractéristique des couches supérieures.

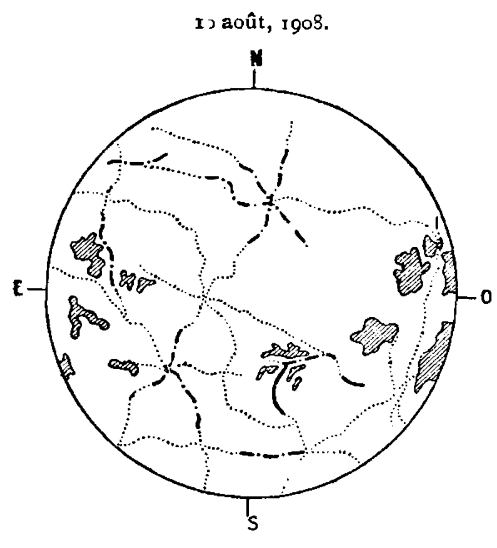

3o septembre, rgo8.

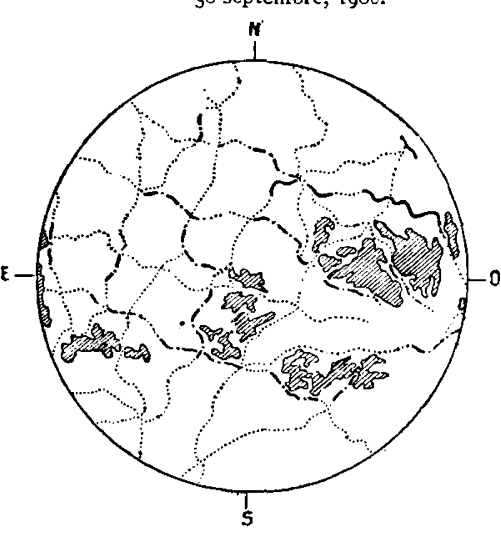

Fic. 3.-Réseau d'alignements relevé dans la couche supérieure de l'atmosphère solaire. Les traits noirs pleins correspondent aux alignements noirs, continues et très nets, appelés filaments: les traits discontinus aux alignements simlaires moins nets, et les traits pointillés aux alignements encore moins visibles et parfois discontinus. Les parties hachees sont les plages brillantes faculaires les plus larges.

Le filament a la même importance que la tache de la surface; il persiste, comme elle, pendant plusieurs rotations et, comme elle aussi, il est le siége de perturbations spéciales, et est accompagné de proéminences.

Dans une première étude j'ai assimilé les taches aux dépressions ou cyclones de notre atmosphère, et les filaments aux anti-cyclones; mais je reviendrai plus loin sur ce rapprochement, qui sera developpé.

\section{(To be continued.)}

\section{UNIVERSITY AND EDUCATIONAL INTELLIGENCE}

Cambridge. - The degree committee of the special board for biology and geology has co-opted Dr. Barclay-Smith and Mr. R. H. Rastall as additional members of the committee.

The special board for physics and chemistry has appointed Mr. C. T. Heycock as assessor in chemistry to the examiners for the Mechanical Sciences Tripos in IgII.

Mr. George Winfield has been elected to the Benn W. Levy studentship.

NO. 2 I 52 , VOL. 85$]$
The director of the observatory gives notice that on fine and clear Saturday evenings during the Lent full term celestial objects will be shown through the Northumberland equatorial to any members of the University and their friends who will come to the observatory between 8 and 10.30 p.m.

THE most important resolution of the last Muslim Education Conference, says the Pioneer Mail, related to an appeal to Muslims for a fund to raise the Aligarh College to the status of a Muslim university. The pro moters of the scheme hope that if requisite funds are forthcoming a Muslim appeal for a charter from King George when he goes to India will not fail. His Highness the Aga Khan has given a lakh of rupees, and other important donations are promised.

A cOPY of the report of the president of the Johns Hopkins University, Baltimore, U.S.A., for the year ending August 3I, 1910, has reached us. It contains a brief summary of the principal events in the history of the University during the academic year under review, together with reports by professors and others having charge of the work in the various departments of the University. There seemed, at the date of the president's report, every probability that the University will benefit by the offer of the General Education Board to contribute towards the endowment fund the sum of $50,000 l$., "provided that on or before December $3 \mathbf{I}$, Igro, a supplementary sum of not less than $150,000 l$. shall be contributed to the University in cash or pledged to the same by good and responsible persons in legally valid subscriptions, payable in cash in not more than three equal annual instalments." At the conclusion of his report the president, Prof. Ira Remsen, writes:- "At the time of this printing the signs are most favourable."

The annual report of University College, Reading, presented at the annual court of governors on January $2 \mathrm{I}$, pointed out that the number of students studying for university degrees shows an increase from 95 in the session $1908-9$ to $x 17$ in the session 1909-10. The other students number 1007. There is a deficit on the year's working of $2016 l$. The principal of the college, Mr. W. M. Childs, told the court that two years ago the college had a total indebtedness on the new buildings of nearly $30,000 l$., but owing to munificent anonymous donations last year, amounting to $16,500 l$., that debt has been brought almost to vanishing point. In addition, Lady Wantage has provided a permanent endowment for Wantage Hall, which she presented to the college some years ago, and that will not only help to defray the cost of maintenance and equipment, but will probably provide for scholarships and bursaries, tenable at the college and the hall. Mr. G. W. Palmer has granted the lease of a recreation ground for twenty-one years rent free, and, thanks to Mr. Alfred Palmer, a new hall will soon be opened for women students.

THE annual meeting of the Association of Technical Institutions will be held, by the courtesy of the Stationers' Company, at their hall on Friday and Saturday, February Io and 11 . The company are generously entertaining the members of the association and a few special guests to luncheon on the Fridity. In the afternoon a vote of thanks will be accorded to Dr. R. T. Glazebrook, F.R.S., the retiring president, for his services during the year I910, and Dr. Glazebrook will move "that Sir Henry F. 